\title{
Correct Me if I'm Wrong, but Do It Right: Error Correction and Learner Uptake in University-level EFL Classrooms
}

\begin{abstract}
By Jelena Milicev ${ }^{*}$
This paper presents the findings on corrective feedback and learner uptake in six university-level EFL classrooms. The study was undertaken by means of observation of ten hours of classroom interaction, five at A1 and another five at B2 level. The classroom observation instrument, developed for the purpose of this research, comprised seven categories for error correction and five for learner uptake. The instrument was used to record the frequency of different types of error correction and learner uptake, as well as the patterns in their co-occurrence. The results show that explicit correction is the predominant type of corrective feedback, to which students normally respond with repetition of the correct form. This tendency is particularly salient in A1 classes, whereas B2 teachers tend to favour recasts, although they do not typically generate learner uptake. Other error correction methods like prompts, explanations, questions, disapproval and error repetition were used less frequently, in spite of the fact that they were highly effective at generating learner uptake and student self-repair.
\end{abstract}

\section{Introduction}

A significant portion of modern EFL instruction seems to be based on communicative and content-based teaching. Research shows that while such an approach leads to the increase in communicative confidence, its focus on content rather than form can diminish grammatical and lexical accuracy (Harley \& Swain, 1984; Lightbown \& Spada, 1990; 2006). Selinker (1972), on the other hand, believes that such inaccuracy is an inevitable part of the learning process, especially in the intermediate stage of L2 development, which bears influences of both L1 and L2, and which he sees as an independent linguistic system called Interlanguage. Allen et al. (1990), in turn, stress the importance of consistent feedback from teachers and peers, while Brown and Rodgers (2009) argue that most learners and teachers feel that it is the teacher's responsibility to provide corrective feedback and help the learners eradicate errors. Chaudron (1977, p.31) defines corrective feedback as 'any reaction of the teacher which clearly transforms, disapprovingly refers to, or demands improvements of the learners' utterance'. Most authors agree that handling

*Independent Researcher, Edinburgh, UK. 
corrective feedback in practice is a challenging task: Allen et al. (1990) point out that if teachers do correct errors, they risk interrupting the communication flow, whereas if they do not correct them, opportunities for students to make links between form and function are reduced. Consequently, Schmitt (2011) argues that when learners are able to get the meaning across using inaccurate language, there may be little motivation to move beyond the current level of language use.

Research into error correction often results in disheartening conclusions about the actual teaching practice. Allen et al. (1990) found that the French immersion teachers they observed corrected only a small number of errors and that they did it in a 'confusing and unsystematic way'. Lyster \& Ranta (1997) point out that the objective of all error correction should be for learners to selfcorrect. Nevertheless, their observation of French immersion classes at the primary level showed an overwhelming tendency for teachers to use recasts, which had no success at generating student self-correction. Yucel's (2000) findings show that teachers and students have different preferences in error correction. He observed the error correction patterns of the teachers of preparatory English classes at a university in Ankara and administered a questionnaire on error correction preferences to 84 students in these courses. The collected data show that explanations, the corrective feedback of choice for most learners, were not used in class at all. Finally, comparing a group that was provided with constant corrective feedback to the one with no corrective feedback at all, DeKeyser (1993) concluded that error correction did benefit low extrinsic motivation students but had no overall effect on student proficiency in the L2.

\section{Background and Purpose of the Study}

The above overview poses a question: is error correction an intrinsically confusing, unsystematic, ineffective, unpopular and ultimately insignificant aspect of teaching as research renders it, or is it in teachers' power to make it work better? Teachers have their own beliefs about corrective feedback, which inform their teaching practice on both conscious and sub-conscious level. It seems crucial, thus, that these beliefs are rooted not only in their own experience as learners or their ideas of what makes a comfortable communicative situation in the classroom, but primarily in the awareness of what makes for effective corrective feedback. Ellis (1997) and Lyster \& Ranta (1997) highlight the didactic potential of error-making, pointing out that teachers should aim for students to self-correct or peer-correct rather than repeat correct forms after the teacher. Lyster \& Ranta's (1997) study of error correction in six French immersion classrooms at primary level indicates that metalinguistic feedback (comments, information or questions), elicitation, clarification requests and repetition of student error often lead to studentgenerated repair, but are used less frequently than recasts, which never produce student-generated repair. 
This study proposes to look into error correction and learner uptake in a different educational context ${ }^{1}$. It records the different types of corrective feedback and learner uptake in six university-level EFL classrooms in Spain. In addition, it examines how the patterns of error correction and learner uptake vary depending on the level of L2 proficiency. It uses classroom observation to find answers to the following research questions:

1. What are the prevalent types of corrective feedback in universitylevel EFL classrooms?

2. Which of these types are successful at eliciting learner uptake and error repair?

3. How do the patterns of error correction and learner uptake vary with the level of L2 proficiency?

\section{Database}

The presented data is derived from classroom observation of error correction and learner uptake in three A1 and three B2 classrooms ${ }^{2}$ at Universidad Europea de Madrid in Spain. The data was collected in real time, by a single observer, during 10 hours of classroom interaction - five in A1 and another five in B2 classrooms. All A1 classes were taught by non-native teachers, while B2 classes were taught by native English speakers. All classes were taught in English. Class groups were formed by means of an objective placement test and consisted of up to 30 students from various degrees. Attendance varied from 2 to 17. All classes had the same objectives: to develop reading, writing, speaking and listening skills, to enable students to take various subject-matter classes in English, as well as to reach or complete B2 level as a graduation requirement. The nature of classes was communicative, with lots of student talking time. However, A1 classes focused on form more than B2 classes.

\section{Design and Development of the Classroom Observation Instrument}

Classroom observation instrument (Table 1) was developed for the purpose of this study and it contained seven categories for error correction and five for learner uptake. The categories were based on Lyster \& Ranta's (1997) as well as Yucel's (2000) observation instruments, which were combined and adjusted to fit my data:

\footnotetext{
${ }^{1}$ This paper is the product of the author's attempt to understand and improve error correction in her own teaching practice. Consequently, she opted to carry out peer observation of corrective feedback at Universidad Europea de Madrid, where she was teaching at the time.

${ }^{2}$ Levels referred to in the paper correspond to the Common European Framework for Languages. See http://www.coe.int/t/dg4/linguistic/cadre1_en.asp
} 
Explicit correction means that the teacher provides the correct form. Recasts involve the teacher's reformulation of a student's utterance, without the error.

Prompts refer to the instances where the teacher elicits the correct form as a completion of his/her utterance.

Explanation means that the teacher explains the error and guides the student to the correct form.

Questions involve eliciting the correct form by asking different yes/no or content questions.

Disapproval involves verbal or non-verbal indications that there is an error in the student utterance.

Error repetition refers to the teacher's repetition of the error, often using interrogative intonation to highlight it.

The categories for learner uptake were adapted from Lyster \& Ranta (1997), who define learner uptake as the student's utterance that follows the teacher's corrective feedback as a reaction to it. Learner uptake can either result in repair of the error on which the feedback is given, or it can represent an utterance that needs further repair. There is no uptake if students do not respond to the correction in any way. Types of uptake are as follows:

Repetition refers to a student's repetition of the correct form previously provided by the teacher.

Incorporation means that a student incorporates the correct form provided by the teacher into a longer utterance.

Self-repair implies self-correction by the student who made the error, prompted by feedback which does not provide the correct form itself.

Peer-repair refers to correction provided by a student other than the one who made the error.

Needs repair refers to all student responses that are in need of further repair: repetition of the same error, different error, L1 response etc.

Table 1 shows the actual instrument which was used during all classroom observations. The simplicity of the tallying system and the clarity of the coded categories made the instrument user-friendly, allowing for easy and reliable recording and note taking. Data analysis methodology was partly modelled on Lyster \& Ranta's (1997) analysis of corrective feedback and learner uptake in French immersion classes. 
Table 1. Classroom observation instrument

\begin{tabular}{|c|l|l|l|l|l|l|}
\hline Feedback type & \multicolumn{5}{|c|}{ Learner uptake } & \multirow{2}{*}{$\begin{array}{c}\text { No } \\
\text { uptake }\end{array}$} \\
\hline Explicit & Repetition & Incorporation & $\begin{array}{c}\text { Self- } \\
\text { repair }\end{array}$ & $\begin{array}{c}\text { Peer- } \\
\text { repair }\end{array}$ & $\begin{array}{c}\text { Needs } \\
\text { repair }\end{array}$ & \\
\hline Recast & & & & & & \\
\hline Prompt & & & & & & \\
\hline Explanation & & & & & & \\
\hline Question & & & & & & \\
\hline Disapproval & & & & & & \\
\hline $\begin{array}{c}\text { Error } \\
\text { repetition }\end{array}$ & & & & & & \\
\hline
\end{tabular}

\section{Analysis}

The recorded data accounts for 146 instances of error correction (Table 2) in the six observed EFL classrooms. It shows that there is an overall preference for explicit error correction, which accounts for $44 \%$ of all corrective feedback. Other frequently used methods are recasts $(17 \%)$, questions $(13 \%)$ and prompts $(12 \%)$, while explanations $(8 \%)$, error repetition $(4 \%)$ and disapproval $(2 \%)$ were used less frequently. There were 77 instances of error correction in A1 classrooms. Explicit corrective feedback was used in $57 \%$ of them, while prompts followed with $17 \%$. Questions and explanations were used in $9 \%$ of instances each. Disapproval, recasts and error repetition combine to account for the remaining $8 \%$ of corrections in A1 classrooms. The most remarkable difference between $\mathrm{A} 1$ and $\mathrm{B} 2$ classrooms concerns recasts. While A1 teachers used them in only $3 \%$ of instances, B2 teachers made them their correction method of choice, using them in $33 \%$ of their corrections. Explicit correction, in turn, came second in B2 classrooms, where it represented $31 \%$ of all corrections. B2 teachers used questions (17\%) and error repetition (7\%) more often than A1 teachers, but made less use of prompts (6\%) and explanations $(6 \%)$. The differences in percentages, however, are not as drastic as those concerning recasts. Disapproval was not used in B2 classrooms.

Table 2. Distribution of feedback types

\begin{tabular}{|c|c|c|c|c|c|c|c|}
\hline & Explicit & Recast & Prompt & Explanation & Question & Disapproval & $\begin{array}{c}\text { Error } \\
\text { repetition }\end{array}$ \\
\hline All & 65 & 25 & 17 & 11 & 19 & 3 & 6 \\
$\mathrm{n}=146$ & $44 \%$ & $17 \%$ & $12 \%$ & $8 \%$ & $13 \%$ & $2 \%$ & $4 \%$ \\
\hline $\mathrm{A} 1$ & 44 & 2 & 13 & 7 & 7 & 3 & 1 \\
$\mathrm{n}=77$ & $57 \%$ & $3 \%$ & $17 \%$ & $9 \%$ & $9 \%$ & $4 \%$ & $1 \%$ \\
\hline $\mathrm{B} 2$ & 21 & 23 & 4 & 4 & 12 & 0 & 5 \\
$\mathrm{n}=69$ & $31 \%$ & $33 \%$ & $6 \%$ & $6 \%$ & $17 \%$ & & $7 \%$ \\
\hline
\end{tabular}


Overall, as shown in Table 3, corrective feedback generated learner uptake of some kind (error repair or an utterance in need of further repair) in $73 \%$ of instances and error repair in 55\%. However, both uptake and repair are much higher in A1 classrooms ( $86 \%$ and $68 \%$ respectively) than in B2 classrooms (59\% and $41 \%$ respectively), which calls for an examination of all individual correction types and their efficacy in different level groups.

Table 3. Turns with uptake and error repair

\begin{tabular}{|c|c|c|}
\hline & Student turns with uptake & Student turns with repair \\
\hline All & 107 & 80 \\
$\mathrm{n}=146$ & $73 \%$ & $55 \%$ \\
\hline A1 & 66 & 52 \\
$\mathrm{n}=77$ & $86 \%$ & $68 \%$ \\
\hline B2 & 41 & 28 \\
$\mathrm{n}=69$ & $59 \%$ & $41 \%$ \\
\hline
\end{tabular}

Table 4 shows learner uptake in all six observed classrooms and the numbers tell us that not all types of feedback are equally effective in generating learner uptake. Error repetition and disapproval always generate uptake. In addition, error repetition leads the way in terms of repair $(100 \%)$, while disapproval follows (67\%). Recasts are least likely to result in either uptake (24\%) or repair $(16 \%)$, while other feedback types fall in between. Questions (90\%), prompts (88\%), explicit correction (81\%) and explanations (64\%) are all successful at generating learner uptake, although explicit correction (69\%) is more likely to result in repair than questions (58\%), prompts (47\%) or explanations $(36 \%)$.

Table 4. Overall learner uptake

\begin{tabular}{|c|c|c|c|}
\hline \multirow{2}{*}{$\begin{array}{c}\text { Feedback } \\
(\mathrm{n}=146)\end{array}$} & Repair & Needs repair & \multirow{2}{*}{ No uptake } \\
\cline { 2 - 3 } $\begin{array}{c}\text { Explicit } \\
(\mathrm{n}=65)\end{array}$ & 45 & 8 & 12 \\
\hline $\begin{array}{c}\text { Recast } \\
(\mathrm{n}=25)\end{array}$ & $69 \%$ & $12 \%$ & $19 \%$ \\
\hline $\begin{array}{c}\text { Prompt } \\
(\mathrm{n}=17)\end{array}$ & 4 & 2 & 19 \\
\hline $\begin{array}{c}\text { Explanation } \\
(\mathrm{n}=11)\end{array}$ & $16 \%$ & $8 \%$ & $76 \%$ \\
\hline $\begin{array}{c}\text { Question } \\
(\mathrm{n}=19)\end{array}$ & $47 \%$ & 7 & 2 \\
\hline $\begin{array}{c}\text { Disapproval } \\
(\mathrm{n}=3)\end{array}$ & 11 & 3 & $12 \%$ \\
\hline Error & $58 \%$ & $28 \%$ & $36 \%$ \\
repetition $(\mathrm{n}=6)$ & $67 \%$ & 6 & 2 \\
\hline
\end{tabular}

If we compare A1 to B2 classrooms (Table 5), we shall see that in A1 classrooms, error repetition and disapproval produced uptake every time they were used. Prompts produced uptake $92 \%$ of the time, explicit correction $89 \%$, questions $86 \%$, explanations $71 \%$. Recasts generated no uptake in A1 classrooms. In terms of repair, most successful methods after error repetition 
(100\%) were explicit correction with $78 \%$, disapproval with $67 \%$ and prompts with $61 \%$ success rate. Questions resulted in repair $57 \%$ of the time and explanations $42 \%$.

Results for B2 classrooms do not differ much. Error repetition is, again, $100 \%$ efficient at generating learner uptake, while questions come second with $91 \%$ success. Other types of feedback follow in this order: prompts (75\%), explicit correction $(66 \%)$, explanations $(50 \%)$ and recasts $(26 \%)$. When it comes to repair, the most effective type is again error repetition, with $100 \%$ success rate, while questions, explicit correction, explanations and recasts follow with $58 \%, 52 \%, 25 \%$ and $17 \%$ respectively. While prompts resulted in learner uptake in $75 \%$ of turns, they did not generate error repair in B2 classrooms.

Table 5. A1 vs. B2 learner uptake by feedback type

\begin{tabular}{|c|c|c|c|c|c|c|c|}
\hline \multicolumn{4}{|c|}{ A1 } & \multicolumn{4}{|c|}{ B2 } \\
\hline \multirow[b]{2}{*}{$\begin{array}{l}\text { Feedback } \\
\quad(\mathrm{n}=77)\end{array}$} & \multicolumn{2}{|c|}{ Uptake } & \multirow[b]{2}{*}{$\begin{array}{c}\text { No } \\
\text { uptake }\end{array}$} & \multirow[b]{2}{*}{$\begin{array}{c}\text { Feedback } \\
\quad(n=69)\end{array}$} & \multicolumn{2}{|c|}{ Uptake } & \multirow{2}{*}{$\begin{array}{c}\text { No } \\
\text { Uptake }\end{array}$} \\
\hline & Repair & $\begin{array}{l}\text { Needs } \\
\text { repair }\end{array}$ & & & Repair & $\begin{array}{l}\text { Needs } \\
\text { repair }\end{array}$ & \\
\hline $\begin{array}{c}\text { Explicit } \\
\text { correction } \\
(\mathrm{n}=44)\end{array}$ & $\begin{array}{c}34 \\
78 \%\end{array}$ & $\begin{array}{c}5 \\
11 \%\end{array}$ & $\begin{array}{c}5 \\
11 \%\end{array}$ & $\begin{array}{c}\text { Explicit } \\
\text { correction } \\
(\mathrm{n}=21)\end{array}$ & $\begin{array}{c}11 \\
52 \%\end{array}$ & $\begin{array}{c}3 \\
14 \%\end{array}$ & $\begin{array}{c}7 \\
34 \%\end{array}$ \\
\hline $\begin{array}{l}\text { Recast } \\
(\mathrm{n}=2)\end{array}$ & 0 & 0 & $\begin{array}{c}2 \\
100 \%\end{array}$ & $\begin{array}{l}\text { Recast } \\
(n=23)\end{array}$ & $\begin{array}{c}4 \\
17 \%\end{array}$ & $\begin{array}{c}2 \\
9 \% \\
\end{array}$ & $\begin{array}{c}17 \\
74 \%\end{array}$ \\
\hline $\begin{array}{l}\text { Prompt } \\
(n=13)\end{array}$ & $\begin{array}{c}8 \\
61 \%\end{array}$ & $\begin{array}{c}4 \\
31 \%\end{array}$ & $\begin{array}{c}1 \\
8 \%\end{array}$ & $\begin{array}{l}\text { Prompt } \\
(\mathrm{n}=4)\end{array}$ & 0 & $\begin{array}{c}3 \\
75 \%\end{array}$ & $\begin{array}{c}1 \\
25 \%\end{array}$ \\
\hline $\begin{array}{l}\text { Explanation } \\
(\mathrm{n}=7)\end{array}$ & $\begin{array}{c}3 \\
42 \%\end{array}$ & $\begin{array}{c}2 \\
29 \%\end{array}$ & $\begin{array}{c}2 \\
29 \%\end{array}$ & $\begin{array}{l}\text { Explanation } \\
(\mathrm{n}=4)\end{array}$ & $\begin{array}{c}1 \\
25 \%\end{array}$ & $\begin{array}{c}1 \\
25 \%\end{array}$ & $\begin{array}{c}2 \\
50 \%\end{array}$ \\
\hline $\begin{array}{l}\text { Question } \\
(\mathrm{n}=7)\end{array}$ & $\begin{array}{c}4 \\
57 \%\end{array}$ & $\begin{array}{c}2 \\
29 \%\end{array}$ & $\begin{array}{c}1 \\
14 \%\end{array}$ & $\begin{array}{l}\text { Question } \\
(\mathrm{n}=12)\end{array}$ & $\begin{array}{c}7 \\
58 \%\end{array}$ & $\begin{array}{c}4 \\
33 \%\end{array}$ & $\begin{array}{c}1 \\
9 \%\end{array}$ \\
\hline $\begin{array}{l}\text { Disapproval } \\
(\mathrm{n}=3)\end{array}$ & $\begin{array}{c}2 \\
67 \%\end{array}$ & $\begin{array}{c}1 \\
33 \%\end{array}$ & 0 & $\begin{array}{c}\text { Disapproval } \\
(\mathrm{n}=0)\end{array}$ & 0 & 0 & 0 \\
\hline $\begin{array}{c}\text { Error } \\
\text { repetition } \\
(\mathrm{n}=1)\end{array}$ & $\begin{array}{c}1 \\
100 \%\end{array}$ & 0 & 0 & $\begin{array}{c}\text { Error } \\
\text { repetition } \\
(\mathrm{n}=5)\end{array}$ & $\begin{array}{c}5 \\
100 \%\end{array}$ & 0 & 0 \\
\hline
\end{tabular}

Lyster \& Ranta (1997) warn that not all repairs are equally meaningful in terms of noticing and understanding corrective feedback. They stress that student-generated repair should be the goal of error correction, rather than mere repetition of the correct form provided by the teacher. Consequently, the categories of self- and peer-repair are conflated into the category of 'studentgenerated repair', while repetitions and incorporations are contained in the category of 'repetition'. Table 6 represents the overall ratio between repetitions and student-generated repair. As explicit corrections and recasts provide the correct form, they can only result in repetition, while the types of corrective feedback which do not provide students with correct answers are more likely to result in student-generated repair. In all the observed classrooms, error repetition proved to be the most efficient in this regard, producing studentgenerated repair every time it was used. Disapproval, questions, prompts and explanations follow with $67 \%, 58 \%, 47 \%$ and $36 \%$ success respectively. 
Table 6. Overall error repair

\begin{tabular}{|c|c|c|c|}
\hline & All repairs & $\begin{array}{c}\text { Repetitions (\% of } \\
\text { feedback type) }\end{array}$ & $\begin{array}{c}\text { Student-generated } \\
\text { repairs (\% of feedback } \\
\text { type) }\end{array}$ \\
\hline $\begin{array}{l}\text { Explicit } \\
(n=65)\end{array}$ & $\begin{array}{c}45 \\
69 \%\end{array}$ & $\begin{array}{c}45 \\
69 \%\end{array}$ & 0 \\
\hline Recast $(n=25)$ & $\begin{array}{c}4 \\
16 \%\end{array}$ & $\begin{array}{c}4 \\
16 \%\end{array}$ & 0 \\
\hline $\begin{array}{l}\text { Prompt } \\
(\mathrm{n}=17)\end{array}$ & $\begin{array}{c}8 \\
47 \%\end{array}$ & 0 & $\begin{array}{c}8 \\
47 \%\end{array}$ \\
\hline $\begin{array}{c}\text { Explanation } \\
(\mathrm{n}=11)\end{array}$ & $\begin{array}{c}4 \\
36 \%\end{array}$ & 0 & $\begin{array}{c}4 \\
36 \%\end{array}$ \\
\hline $\begin{array}{l}\text { Question } \\
(\mathrm{n}=19)\end{array}$ & $\begin{array}{c}11 \\
58 \%\end{array}$ & 0 & $\begin{array}{c}11 \\
58 \%\end{array}$ \\
\hline $\begin{array}{c}\text { Disapproval } \\
(n=3)\end{array}$ & $\begin{array}{c}2 \\
67 \%\end{array}$ & 0 & $\begin{array}{c}2 \\
67 \%\end{array}$ \\
\hline $\begin{array}{l}\text { Error } \\
\text { repetition } \\
(n=6)\end{array}$ & $\begin{array}{c}6 \\
100 \%\end{array}$ & 0 & $\begin{array}{c}6 \\
100 \%\end{array}$ \\
\hline
\end{tabular}

Comparing A1 to B2 student-generated repair (Table 7), we can see that error repetition is equally effective $(100 \%)$ in both group levels. In A1 classrooms, disapproval (67\%), prompts (61\%), questions $(57 \%)$, and explanations (43\%) follow. Explicit corrections and recasts have no success in eliciting student-generated repair in any classrooms. In B2 classrooms, apart from error repetition, only questions $(58 \%)$ and explanations $(25 \%)$ were able to produce student-generated repair.

Table 7. A1 vs. B2 error repair

\begin{tabular}{|c|c|c|c|c|c|c|c|}
\hline \multicolumn{4}{|c|}{ A1 } & \multicolumn{4}{|c|}{ B2 } \\
\hline $\begin{array}{l}\text { Feedback } \\
\quad(\mathrm{n}=77)\end{array}$ & $\begin{array}{l}\text { All } \\
\text { repairs }\end{array}$ & $\begin{array}{l}\text { Repetition } \\
\text { (\% of } \\
\text { feedback } \\
\text { type) }\end{array}$ & $\begin{array}{c}\text { Student } \\
\text { repair } \\
\text { (\% of } \\
\text { feedback } \\
\text { type) }\end{array}$ & $\begin{array}{l}\text { Feedback } \\
\quad(n=69)\end{array}$ & $\begin{array}{l}\text { All } \\
\text { repairs }\end{array}$ & $\begin{array}{l}\text { Repetition } \\
\text { (\% of } \\
\text { feedback } \\
\text { type) }\end{array}$ & $\begin{array}{l}\text { Student } \\
\text { repair (\% } \\
\text { of } \\
\text { feedback } \\
\text { type) }\end{array}$ \\
\hline $\begin{array}{l}\text { Explicit } \\
(\mathrm{n}=44)\end{array}$ & $\begin{array}{c}34 \\
77 \%\end{array}$ & $\begin{array}{c}34 \\
77 \%\end{array}$ & 0 & $\begin{array}{l}\text { Explicit } \\
(\mathrm{n}=21)\end{array}$ & $\begin{array}{c}11 \\
52 \%\end{array}$ & $\begin{array}{c}11 \\
52 \%\end{array}$ & 0 \\
\hline $\begin{array}{c}\text { Recast } \\
(\mathrm{n}=2)\end{array}$ & 0 & 0 & 0 & $\begin{array}{l}\text { Recast } \\
(\mathrm{n}=23)\end{array}$ & $\begin{array}{c}4 \\
17 \% \\
\end{array}$ & $\begin{array}{c}4 \\
17 \% \\
\end{array}$ & 0 \\
\hline $\begin{array}{l}\text { Prompt } \\
(\mathrm{n}=13)\end{array}$ & $\begin{array}{c}8 \\
61 \%\end{array}$ & 0 & $\begin{array}{c}8 \\
61 \%\end{array}$ & $\begin{array}{l}\text { Prompt } \\
(\mathrm{n}=4)\end{array}$ & 0 & 0 & 0 \\
\hline $\begin{array}{l}\text { Explanation } \\
(\mathrm{n}=7)\end{array}$ & $\begin{array}{c}3 \\
43 \% \\
\end{array}$ & 0 & $\begin{array}{c}3 \\
43 \% \\
\end{array}$ & $\begin{array}{c}\text { Explanation } \\
(\mathrm{n}=4)\end{array}$ & $\begin{array}{c}1 \\
25 \%\end{array}$ & 0 & $\begin{array}{c}1 \\
25 \%\end{array}$ \\
\hline $\begin{array}{l}\text { Question } \\
(\mathrm{n}=7)\end{array}$ & $\begin{array}{c}4 \\
57 \% \\
\end{array}$ & 0 & $\begin{array}{c}4 \\
57 \% \\
\end{array}$ & $\begin{array}{l}\text { Question } \\
(\mathrm{n}=12)\end{array}$ & $\begin{array}{c}7 \\
58 \% \\
\end{array}$ & 0 & $\begin{array}{c}7 \\
58 \% \\
\end{array}$ \\
\hline $\begin{array}{c}\text { Disapproval } \\
(\mathrm{n}=3)\end{array}$ & $\begin{array}{c}2 \\
67 \% \\
\end{array}$ & 0 & $\begin{array}{c}2 \\
67 \% \\
\end{array}$ & $\begin{array}{c}\text { Disapproval } \\
(\mathrm{n}=0)\end{array}$ & 0 & 0 & 0 \\
\hline $\begin{array}{c}\text { Error } \\
\text { repetition } \\
(\mathrm{n}=1)\end{array}$ & $\begin{array}{c}1 \\
100 \%\end{array}$ & 0 & $\begin{array}{c}1 \\
100 \%\end{array}$ & $\begin{array}{c}\text { Error } \\
\text { repetition } \\
(\mathrm{n}=5)\end{array}$ & $\begin{array}{c}5 \\
100 \%\end{array}$ & 0 & $\begin{array}{c}5 \\
100 \%\end{array}$ \\
\hline
\end{tabular}


In terms of repair, explicit correction (56\%) accounts for the highest percentage across all observed groups (Table 8). However, the feedback types which produce student-generated repair are questions (36\%), prompts $(26 \%)$, error repetition (19\%), explanations $(13 \%)$ and disapproval (6\%). Explicit correction and recasts do not result in student-generated repair.

Table 8. Overall repair by each feedback type

\begin{tabular}{|c|c|c|c|c|c|c|c|}
\hline & Explicit & Recast & Prompt & Explanation & Question & Disapproval & $\begin{array}{c}\text { Error } \\
\text { repetition }\end{array}$ \\
\hline $\begin{array}{c}\text { All } \\
\text { repairs } \\
(\mathrm{n}=80)\end{array}$ & $\begin{array}{c}45 \\
56 \%\end{array}$ & $\begin{array}{c}4 \\
5 \%\end{array}$ & $\begin{array}{c}8 \\
10 \%\end{array}$ & $\begin{array}{c}4 \\
5 \%\end{array}$ & $\begin{array}{c}11 \\
14 \%\end{array}$ & $\begin{array}{c}2 \\
2.5 \%\end{array}$ & $\begin{array}{c}6 \\
7.5 \%\end{array}$ \\
\hline $\begin{array}{l}\text { Student- } \\
\text { generated } \\
\text { repairs } \\
(\mathrm{n}=31)\end{array}$ & 0 & 0 & $\begin{array}{c}8 \\
26 \%\end{array}$ & $\begin{array}{c}4 \\
13 \%\end{array}$ & $\begin{array}{c}11 \\
36 \%\end{array}$ & $\begin{array}{c}2 \\
6 \%\end{array}$ & $\begin{array}{c}6 \\
19 \%\end{array}$ \\
\hline
\end{tabular}

When we compare error repair in A1 and B2 classrooms (Table 9), we see that explicit correction accounts for the highest number of repairs in both level groups (65\% and 39\% respectively). However, these groups differ in terms of student-generated repair. Prompts (44\%) and questions (22\%) work best in A1 classrooms, while questions $(54 \%)$ and error repetition $(38 \%)$ are responsible for the highest rates of student-generated repair in B2 classrooms. Although very efficient in A1 classrooms, prompts did not retrieve any repairs in B2 classrooms.

Table 9. A1 vs. B2 repairs attributed to each feedback type

\begin{tabular}{|c|c|c|c|c|c|c|c|}
\hline \multicolumn{7}{|c|}{ A1 } \\
\hline & Explicit & Recast & Prompt & Explanation & Question & Disapproval & $\begin{array}{c}\text { Error } \\
\text { repetition }\end{array}$ \\
\hline $\begin{array}{c}\text { All } \\
\text { repairs } \\
\text { n=52 }\end{array}$ & $\begin{array}{c}34 \\
65 \%\end{array}$ & 0 & $\begin{array}{c}8 \\
15 \%\end{array}$ & $\begin{array}{c}3 \\
6 \%\end{array}$ & $\begin{array}{c}4 \\
8 \%\end{array}$ & $\begin{array}{c}2 \\
4 \%\end{array}$ & $\begin{array}{c}1 \\
2 \%\end{array}$ \\
\hline $\begin{array}{c}\text { Student- } \\
\text { generated } \\
\text { repairs } \\
\text { n=18 }\end{array}$ & 0 & 0 & $\begin{array}{c}8 \\
44 \%\end{array}$ & $\begin{array}{c}3 \\
17 \%\end{array}$ & $\begin{array}{c}4 \\
22 \%\end{array}$ & $\begin{array}{c}2 \\
11 \%\end{array}$ & $\begin{array}{c}1 \\
6 \%\end{array}$ \\
\hline \multicolumn{1}{|c|}{ B2 } & Recast & Prompt & Explanation & Question & Disapproval & $\begin{array}{c}\text { Error } \\
\text { repetition }\end{array}$ \\
\hline $\begin{array}{c}\text { All } \\
\text { repairs } \\
\text { n=28 }\end{array}$ & $39 \%$ & $14 \%$ & 0 & $\begin{array}{c}1 \\
4 \%\end{array}$ & $\begin{array}{c}7 \\
25 \%\end{array}$ & 0 & $\begin{array}{c}5 \\
18 \%\end{array}$ \\
\hline $\begin{array}{c}\text { Student- } \\
\text { generated } \\
\text { repairs } \\
\text { n=13 }\end{array}$ & 0 & 0 & 0 & $\begin{array}{c}1 \\
8 \%\end{array}$ & $\begin{array}{c}7 \\
54 \%\end{array}$ & 0 & $\begin{array}{c}5 \\
38 \%\end{array}$ \\
\hline
\end{tabular}

As we have seen earlier (Table 3), A1 groups have higher rates of uptake and repair than B2 groups. Nevertheless, only 35\% of all A1 repairs are student-generated, compared to $46 \%$ of B2 repairs (Table 10). 
Table 10. Student-generated repair

\begin{tabular}{|c|c|}
\hline $\begin{array}{c}\text { Turns } \\
\text { with repair }\end{array}$ & $\begin{array}{c}\text { Turns with } \\
\text { student-generated repair }\end{array}$ \\
\hline All & 31 \\
$\mathrm{n}=80$ & $39 \%$ \\
\hline $\mathrm{A} 1$ & 18 \\
$\mathrm{n}=52$ & $35 \%$ \\
\hline $\mathrm{B} 2$ & 13 \\
$\mathrm{n}=28$ & $46 \%$ \\
\hline
\end{tabular}

In order to account for this difference, we shall look into learner response to specific types of corrective feedback. First, we shall distinguish between feedback types that provide students with the correct form (explicit correction and recasts) and those that do not (prompts, explanations, questions, disapproval and error repetition). To this end, the former types will be conflated into the category of 'explicit correction', while the latter will be referred to as 'non-explicit correction'. Table 11 shows that both level groups have similar ratios of explicit and non-explicit correction, with the B2 percentage of explicit feedback $(64 \%)$ being even slightly higher than A1 $(60 \%)$.

Table 11. A1 vs. B2 learner uptake (\% of explicit and non-explicit correction)

\begin{tabular}{|c|c|c|c|}
\hline $\begin{array}{c}\text { A1 Feedback type } \\
(\mathrm{n}=77)\end{array}$ & $\begin{array}{c}\text { A1 Learner } \\
\text { uptake }\end{array}$ & $\begin{array}{c}\text { B2 Feedback type } \\
(\mathrm{n}=69)\end{array}$ & $\begin{array}{c}\text { B2 Learner } \\
\text { uptake }\end{array}$ \\
\hline $\begin{array}{c}\text { Explicit correction } \\
46\end{array}$ & 39 & $\begin{array}{c}\text { Explicit correction } \\
44\end{array}$ & 20 \\
$(60 \%)$ & $(85 \%)$ & $(64 \%)$ & $(45 \%)$ \\
\hline $\begin{array}{c}\text { Non-explicit } \\
\text { correction }\end{array}$ & & $\begin{array}{c}\text { Non-explicit } \\
\text { correction }\end{array}$ & \\
31 & 27 & 25 & 21 \\
$(40 \%)$ & $(87 \%)$ & $(36 \%)$ & $(84 \%)$ \\
\hline
\end{tabular}

The difference in student-generated repair between the two group levels is to do with how these groups respond to 'explicit correction'. A1 groups responded to $85 \%$ of such feedback, which predisposed their repair to qualify as 'repetition' rather than 'student-generated'. On the other hand, B2 students responded to only $45 \%$ of explicit corrections, which increased their potential for student-generated repair over repetitions. What caused B2 students to respond in this way? They have higher level of L2 proficiency and more experience in English language learning. These two variables seem to account for more advanced learning strategies, which help learners discriminate between repetitive and more meaningful tasks and engage in the latter rather than former. Therefore, the quality of learner uptake and error repair seems to be influenced by level-dependant learner judgment as much as by teacher practice. Learning strategies behind learner uptake and error repair should be the object of further study. 


\section{Conclusions}

Analysis of the above data allows me to answer my research questions in the following way:

1. The prevalent types of corrective feedback in university-level EFL classrooms are explicit correction (44\%) and the recast (17\%). Teachers also use questions (13\%), prompts (12\%), explanations $(8 \%)$, error repetition (4\%) and disapproval (2\%) to elicit error correction.

2. The type of feedback affects the quality of learner uptake. Overall, most feedback types are successful at generating learner uptake and they do it at the following rates: error repetition (100\%), disapproval (100\%), questions (90\%), prompts (88\%), explicit correction (81\%) and explanations $(64 \%)$. The least likely to generate uptake is the recast, with only $24 \%$ success. Error repair is generated most successfully by error repetition (100\%), explicit correction (69\%), disapproval (67\%), questions (58\%), prompts (47\%) and explanations (36\%), while recasts generate repair only in $16 \%$ of instances. Student-generated repair is most likely to be produced by error repetition (100\%), disapproval $(67 \%)$, questions $(58 \%)$, prompts $(47 \%)$ and explanations $(36 \%)$, but cannot be generated by explicit correction and recasts, as they already contain the correct form. Nevertheless, some of the most successful feedback types, like error repetition and disapproval, were seldom used, and are therefore responsible for a comparatively small number of repairs.

3. Patterns in error correction and learner uptake vary with level of L2 proficiency in the following ways:

Firstly, different feedback types dominate different level groups. While explicit correction is typical for the more form-focused A1 lessons, recasts are ubiquitous in the message-focused B2 classes. These patterns imply that class level and focus can influence the choice of error correction methods, as teachers seem to opt for explicit correction to provide more help and guidance in the form-focused beginners' classes, and for recasts to provide feedback that allows for an uninterrupted communication flow in the message-oriented higher level groups.

Secondly, when it comes to the efficacy of different feedback types in generating uptake and repair, the two levels differ to an extent. Error repetition, prompts, questions and explicit correction are successful in generating learner uptake in both level groups, while explanations generate more uptake and repair in A1 than in B2 groups. Error repetition, explicit correction and questions favour repairs in both level groups, while prompts and disapproval favour only those in A1. When it comes to student-generated repair, interrogative types of feedback like error repetition and questions are efficient in all classrooms. In addition, A1 groups also benefit from disapproval and prompts. 
Thirdly, the level of L2 proficiency has an effect on the quality of learner uptake and error repair. Namely, A1 classes have a significantly higher rate of learner uptake and error repair. This might be to do with the conversational nature of B2 classes, whose focus on message rather than form might cause the students to ignore some of the feedback on form. However, B2 classes have a significantly higher percentage of student-generated repairs. This might be caused by the fact that B2 classes seem to choose to respond mostly to the feedback that does not contain the correct form itself, which automatically increases their chances of scoring highly in student-generated repair. Such choice indicates advanced learning strategies likely to be found in experienced language learners, and implies that the quality of error repair is, to an extent, level-dependant.

Overall, error repair occurred in more than half of the corrections $(55 \%)$, but only $21 \%$ of them resulted in student-generated repair. An informed and systematic use of suitable corrective feedback types in different teaching contexts can help teachers improve their learners' error repair rate. Awareness of each type's limitations as well as its potential for generating student selfcorrection is crucial.

\section{References}

Allen, P. et al., 1990. Aspects of classroom treatment: Toward a more comprehensive view of second language education. In: B. Harley, et al., eds. The development of second language proficiency (pp. 57-81). Cambridge: Cambridge University Press.

Brown, J.D. \& Rodgers, T. S., 2009. Doing Second Language Research. Oxford: Oxford University Press

Chaudron, C., 1977. A descriptive model of discourse in the corrective treatment of learners' errors. Language learning, 27, pp.29-46

DeKeyser, R., 1993. The effect of error correction on L2 grammar knowledge and oral proficiency. Modern Language Journal, 77, pp.501-514

Ellis, R., 1997. Second language acquisition. Oxford: Oxford University Press

Harley, B., \& Swain, M., 1984. The Interlanguage of Immersion Students and Its implications for Second Language Teaching. In: A. Davies, C. Criper, \& A.P.R. Howatt, eds. Interlanguage. Edinburgh: Edinburgh University Press; pp.291-311.

Lightbown, P. \& Spada, N., 1990. Focus on form and corrective feedback in communicative language teaching: effects on second language learning. Studies in Second Language Acquisition, 12, pp.429-448

Lightbown, P. \& Spada, N., 2006. How Languages are Learnt. Oxford: Oxford University Press

Lyster, R. \& Ranta, L., 1997. Corrective feedback and learner uptake: Negotiation of form in communicative classrooms. Studies in Second Language Acquisition 19(1), pp.37-61.

Schmitt, N., 2011. An Introduction to Applied Linguistics. 2nd ed., London: Hodder Education

Selinker, L., 1972. Interlanguage. International Review of Applied Linguistics 10(2), pp.209-31 
Yucel, S., 2000. Snapshots of classroom interaction: Teacher question behaviour and error correction strategies. In: S. Tuzel-Koymen \& E.Kortan, eds. Interaction on the threshold of a new millennium. Ankara: Middle Eastern Technical University. pp.144-54. 
